\title{
On the Elementary Solution of the Operator $\circledast_{B}^{k}$
}

\author{
Somboon Niyom \\ Department of Mathematics, Chiangmai University \\ Chiangmai, 50200, THAILAND \\ E-mail: sbniyom@hotmail.com \\ Amnuay Kananthai (Corresponding author) \\ Department of Mathematics, Chiangmai University \\ Chiangmai, 50200, THAILAND \\ E-mail: malamnka@science.cmu.ac.th
}

The research is financed by Office of the Higher Education Commission, Thailand(Sponsoring information)

\section{Abstract}

In this paper, we study the elementary solution of the operator $\circledast_{B}^{k}$ which is defined by

$$
\circledast_{B}^{k}=\left[\left(B_{x_{1}}+B_{x_{2}}+\cdots+B_{x_{p}}\right)^{3}+\left(B_{x_{p+1}}+\cdots+B_{x_{p+q}}\right)^{3}\right]^{k}
$$

where $p+q=n$ is the dimension of $\mathbb{R}_{n}^{+}=\left\{\left(x=x_{1}, x_{2}, \ldots, x_{n}\right): x_{1}>0, x_{2}>0, \ldots, x_{n}>0\right\}, B_{x_{i}}=\frac{\partial^{2}}{\partial x_{i}^{2}}+\frac{2 v_{i}}{x_{i}} \frac{\partial}{\partial x_{i}}$, $2 v_{i}=2 \alpha_{i}+1, \alpha_{i}>-\frac{1}{2}, x_{i}>0, i=1,2, \ldots, n$ and $k$ is a positive integer. After that, we apply such an elementary solution to solve the equation $\circledast_{B}^{k} u(x)=f(x)$, where $f$ is a generalized function and $u$ is an unknown function.

Keywords: Dirac delta distribution, Tempered distribution, Fourier-Bessel transform, Bessel operator

\section{Introduction}

I. M. Gelfand and G. E. Shilov (1964) have first introduced the elementary solution of the $n$-dimensional classical diamond operator. S. E. Trione has shown that the $n$-dimensional ultra-hyperbolic equation has $u(x)=R_{2 k}(x)$ as unique elementary solution. Later, M. A. Tellez has proved that $R_{2 k}(x)$ exists only for case $p$ is odd with $p+q=n$. A. Kananthai has showed that the solution in the convolution form $u(x)=(-1)^{k} S_{2 k}(x) * R_{2 k}(x)$ is the unique elementary solution of the $\nabla^{k} u(x)=\delta$. Furthermore, M. Z. Sarikaya and H. Yildirim have introduced the Bessel diamond operator and have proved that the convolution solution $u(x)=(-1)^{k} S_{2 k}(x) * R_{2 k}(x)$ is the unique elementary solution of the $\diamond_{B}^{k} u(x)=\delta$, where $\diamond_{B}^{k}$ is the Bessel diamond operator iterated $k$ times with $x \in \mathbb{R}_{n}^{+}$,

$$
\diamond_{B}^{k}=\left[\left(B_{x_{1}}+B_{x_{2}}+\cdots+B_{x_{p}}\right)^{2}-\left(B_{x_{p+1}}+\cdots+B_{x_{p+q}}\right)^{2}\right]^{k}, p+q=n .
$$

The Bessel diamond operator can be expressed in the form $\diamond_{B}=\square_{B} \triangle_{B}=\triangle_{B} \square_{B}$, where $\triangle_{B}$ is the Laplace-Bessel operator which is defined by

$$
\triangle_{B}=B_{x_{1}}+B_{x_{2}}+\cdots+B_{x_{n}}
$$

and $\square_{B}$ is the Bessel ultra-hyperbolic operator which is defined by

$$
\square_{B}=B_{x_{1}}+B_{x_{2}}+\cdots+B_{x_{p}}-B_{x_{p+1}}-B_{x_{p+2}}-\cdots-B_{x_{p+q}}
$$

In this paper, at first we study the elementary solution of the $\circledast_{B}^{k}$ operator, that is

$$
\circledast_{B}^{k} G(x)=\delta,
$$

where $G(x)$ is the elementary solution of such equation, $\delta$ is the Dirac delta distribution, $k$ is nonnegative integer and the 
$\circledast_{B}$ operator is defined by

$$
\begin{aligned}
\circledast_{B} & =\left(\sum_{i=1}^{p} B_{x_{i}}\right)^{3}+\left(\sum_{i=p+1}^{p+q} B_{x_{i}}\right)^{3} \\
& =\left[\sum_{i=1}^{p} B_{x_{i}}+\sum_{i=p+1}^{p+q} B_{x_{i}}\right]\left[\left(\sum_{i=1}^{p} B_{x_{i}}\right)^{2}-\sum_{i=1}^{p} B_{x_{i}} \sum_{i=p+1}^{p+q} B_{x_{i}}+\left(\sum_{i=p+1}^{p+q} B_{x_{i}}\right)^{2}\right] \\
& =\triangle_{B}\left[\triangle_{B}^{2}-\frac{3}{4}\left(\triangle_{B}+\square_{B}\right)\left(\triangle_{B}-\square_{B}\right)\right] \\
& =\frac{3}{4} \triangle_{B} \square_{B}^{2}+\frac{1}{4} \triangle_{B}^{3} \\
& =\frac{3}{4} \triangleright_{B} \square_{B}+\frac{1}{4} \triangle_{B}^{3} .
\end{aligned}
$$

After that, we apply such an elementary solution to solve for the solution of the equation $\circledast_{B}^{k} G(x)=f(x)$, where $f(x)$ is a generalized function and $u(x)$ is an unknown function for $x \in \mathbb{R}_{n}^{+}$.

\section{Preliminaries}

The generalized shift operator, $T_{x}^{y}$ has the following form (B.M. Levitan, 1951, p.102-143),

$$
T_{x}^{y}=C_{v}^{*} \int_{0}^{\pi} \cdots \int_{0}^{\pi} \varphi\left(s_{1}, \ldots, s_{n}\right)\left(\prod_{i=1}^{n} \sin ^{2 v_{i}-1} \theta_{i}\right) d \theta_{1} \cdots d \theta_{n},
$$

where $s_{i}^{2}=x_{i}^{2}+y_{i}^{2}-2 x_{i} y_{i} \cos \theta_{i}, x, y \in \mathbb{R}_{n}^{+}$and $C_{v}^{*}=\prod_{i=1}^{n} \frac{\Gamma\left(v_{i}+1\right)}{\Gamma\left(\frac{1}{2}\right) \Gamma\left(v_{i}\right)}$. We remark that this shift operator is closely connected with the Bessel differential operator (B.M. Levitan, 1951, p.102-143),

$$
\begin{aligned}
\frac{d^{2} \varphi}{d x_{i}^{2}}+\frac{2 v_{i}}{x_{i}} \frac{d \varphi}{d x_{i}} & =\frac{d^{2} \varphi}{d y_{i}^{2}}+\frac{2 v_{i}}{y_{i}} \frac{d \varphi}{d y_{i}}, \\
\varphi\left(x_{i}, 0\right) & =f(x), \\
\varphi_{y_{i}}\left(x_{i}, 0\right) & =0,
\end{aligned}
$$

where $x_{i}, y_{i} \in \mathbb{R}_{n}^{+}$for $i=1,2, \ldots, n$. The convolution operator denoted by $T_{x}^{y}$ is defined as follows

$$
(f * \varphi)(x)=\int_{\mathbb{R}_{n}^{+}} f(y) T_{x}^{y} \varphi(x)\left(\prod_{i=1}^{n} y_{i}^{2 v_{i}}\right) d y .
$$

Convolution in (6) is known as a $B$-convolution. We note the following properties of the $B$-convolution and the generalized shift operator,

(a) $T_{x}^{y} \cdot 1=1$.

(b) $T_{x}^{0} \cdot f(x)=f(x)$.

(c) If $f(x), g(x) \in C\left(\mathbb{R}_{n}^{+}\right), g(x)$ is a bounded function for $x \in \mathbb{R}_{n}^{+}$and

$$
\int_{\mathbb{R}_{n}^{+}}|f(x)|\left(\prod_{i=1}^{n} x_{i}^{2 v_{i}}\right) d x<\infty
$$

then

$$
\int_{\mathbb{R}_{n}^{+}} T_{x}^{y} f(x) g(y)\left(\prod_{i=1}^{n} y_{i}^{2 v_{i}}\right) d y=\int_{\mathbb{R}_{n}^{+}} f(y) T_{x}^{y} g(x)\left(\prod_{i=1}^{n} y_{i}^{2 v_{i}}\right) d y .
$$

(d) From (c), we have the following equality for $g(x)=1$,

$$
\int_{\mathbb{R}_{n}^{+}} T_{x}^{y} f(x)\left(\prod_{i=1}^{n} y_{i}^{2 v_{i}}\right) d y=\int_{\mathbb{R}_{n}^{+}} f(y)\left(\prod_{i=1}^{n} y_{i}^{2 v_{i}}\right) d y .
$$

(e) $(f * g)(x)=(g * f)(x)$. 
The Fourier-Bessel transformation and its inverse transformation are defined as follows (H. Yildirim, 1995),

$$
\begin{gathered}
\left(F_{B} f\right)(x)=C_{v} \int_{\mathbb{R}_{n}^{+}} f(y)\left(\prod_{i=1}^{n} J_{v_{i}-\frac{1}{2}}\left(x_{i} y_{i}\right) y_{i}^{2 v_{i}}\right) d y \\
\left(F_{B}^{-1} f\right)(x)=\left(F_{B} f\right)(-x), C_{v}=\left(\prod_{i=1}^{n} 2^{v_{i}-\frac{1}{2}} \Gamma\left(v_{i}+\frac{1}{2}\right)\right)^{-1},
\end{gathered}
$$

where $J_{v_{i}-\frac{1}{2}}\left(x_{i} y_{i}\right)$ is the normalized Bessel function which is the eigenfunction of the Bessel differential operator. There are following equalities for Fourier-Bessel transformation (H. Yildirim, 1995),

$$
F_{B} \delta(x)=1 \text { and } F_{B}(f * g)(x)=F_{B} f(x) \cdot F_{B} g(x) .
$$

Lemma 1. There is a following equality for Fourier-Bessel transformation

$$
F_{B}\left(|x|^{-\alpha}\right)=2^{n+2|v|-2 \alpha} \Gamma\left(\frac{n+2|v|-\alpha}{2}\right)\left[\Gamma\left(\frac{\alpha}{2}\right)\right]^{-1}|x|^{\alpha-n-2|v|},
$$

where $|v|=v_{1}+v_{2}+\cdots+v_{n}$.

Proof. (H. Yildirim, 1995).

Lemma 2. Given the equation $\triangle_{B}^{k} u(x)=\delta(x)$ for $x \in \mathbb{R}_{n}^{+}$, where $\triangle_{B}^{k}$ is the Laplace-Bessel operator iterated $k$-times defined by (2). Then $u(x)=(-1)^{k} S_{2 k}(x)$ is an elementary solution of the $\triangle_{B}^{k}$ operator, where

$$
S_{2 k}(x)=\frac{2^{n+2|v|-4 k} \Gamma\left(\frac{n+2|v|-2 k}{2}\right)}{\prod_{i=1}^{n} 2^{v_{i}-\frac{1}{2}} \Gamma\left(v_{i}+\frac{1}{2}\right) \Gamma(k)}|x|^{2 k-n-2|v|} .
$$

Proof. (H. Yildirim, 1995).

Lemma 3. Given the equation $\square_{B}^{k} u(x)=\delta(x)$ for $x \in \Gamma_{+}=\left\{x \in \mathbb{R}_{n}^{+}: x_{1}>0, x_{2}>0, \cdots, x_{n}>0\right.$ and $\left.V>0\right\}$, where $\square{ }_{B}^{k}$ is the Bessel-ultra-hyperbolic operator iterated $k$-times defined by (3). Then $u(x)=R_{2 k}(x)$ is an elementary solution of the $\square_{B}^{k}$ operator, where

$$
R_{2 k}(x)=\frac{V^{\frac{2 k-n-2|w|}{2}}}{K_{n}(2 k)}
$$

for

and

$$
V=x_{1}^{2}+x_{2}^{2}+\cdots+x_{p}^{2}-x_{p+1}^{2}-\cdots-x_{p+q}^{2}
$$

$$
K_{n}(2 k)=\frac{\pi^{\frac{n+2|v|-1}{2}} \Gamma\left(\frac{2+2 k-n-2|v|}{2}\right) \Gamma\left(\frac{1-2 k}{2}\right) \Gamma(2 k)}{\Gamma\left(\frac{2+2 k-p-2|v|}{2}\right) \Gamma\left(\frac{p-2 k}{2}\right)}
$$

Proof. (H. Yildirim, M. Z. Sarikaya and S. Öztürk, 2004, p.375-387).

Lemma 4. The functions $S_{2 k}(x)$ and $R_{2 k}(x)$ are homogeneous distributions of order $(2 k-n-2|v|)$ for $\operatorname{Re}(2 k)<n+2|v|$. In particular, the B-convolution $S_{2 k}(x) * R_{2 k}(x)$ exists and is a tempered distribution.

Proof. (H. Yildirim, M. Z. Sarikaya and S. Öztürk, 2004, p.375-387).

Lemma 5. Given the equation $\diamond_{B}^{k} u(x)=\delta(x)$ for $x \in \mathbb{R}_{n}^{+}$, where $\diamond_{B}^{k}$ is the diamond Bessel operator iterated $k$-times defined by (1). Then $u(x)=(-1)^{k} S_{2 k}(x) * R_{2 k}(x)$ is an elementary solution of the $\diamond_{B}^{k}$ operator.

Proof. (H. Yildirim, M. Z. Sarikaya and S. Öztürk, 2004, p.375-387).

Lemma 6. Let $k$ and $r$ be nonnegative integer.

(a) Let $S_{2 k}(x)$ and $S_{2 r}(x)$ be defined by (7), then $S_{2 k}(x) * S_{2 r}(x)=S_{2 k+2 r}(x)$.

(b) Let $R_{2 k}(x)$ and $R_{2 r}(x)$ be defined by $(8)$, then $R_{2 k}(x) * R_{2 r}(x)=R_{2 k+2 r}(x)$.

Proof. (M. Z. Sarikaya and H. Yildirim, 2009, p.18-22). 
Lemma 7. The convolution $S_{6 k}(x) * R_{4 k}(x)$ exists and is a tempered distribution where $S_{6 k}(x)=S_{2 k}(x) * S_{2 k}(x) * S_{2 k}(x)$ and $R_{4 k}(x)=R_{2 k}(x) * R_{2 k}(x)$ such that $S_{2 k}(x)$ and $R_{2 k}(x)$ are defined by (7) and (8), respectively.

Proof. Since $S_{2 k}(x) * R_{2 k}(x)$ exists and is a tempered distribution, by W.F. Donoghue (1969, p.156-159), we obtain $S_{6 k}(x) * R_{4 k}(x)$ exists and is a tempered distribution.

Lemma 8. Let $S_{6}(x)$ with $k=3$ and $R_{4}(x)$ with $k=2$ be defined by (7) and (8) respectively. Then

(a) $\diamond_{B} \square_{B}\left(S_{6}(x) * R_{4}(x)\right)=-S_{4}(x)$,

(b) $\triangle_{B}^{3}\left(S_{6}(x) * R_{4}(x)\right)=-R_{4}(x)$.

Proof. (a) We obtain

$$
\begin{aligned}
\diamond_{B} \square_{B}\left(S_{6}(x) * R_{4}(x)\right) & =\diamond_{B} \square_{B}\left((-1)^{2} S_{6}(x) * R_{4}(x)\right) \\
& =\diamond_{B}\left((-1) S_{2}(x) * R_{2}(x)\right) * \square_{B}\left(R_{2}(x)\right) *(-1) S_{4}(x) \\
& =\delta(x) * \delta(x) *(-1) S_{4}(x) \\
& =-S_{4}(x) .
\end{aligned}
$$

(b) We get

$$
\begin{aligned}
\triangle_{B}^{3}\left(S_{6}(x) * R_{4}(x)\right) & =\triangle_{B}^{3}\left((-1)^{4}\left(S_{6}(x) * R_{4}(x)\right)\right. \\
& =\triangle_{B}^{3}\left((-1)^{3} S_{2(3)}(x)\right) *(-1) R_{4}(x) \\
& =\delta(x) *(-1) R_{4}(x) \\
& =-R_{4}(x)
\end{aligned}
$$

\section{Main results}

Theorem 1. Given the equation

$$
\circledast_{B}^{k} G(x)=\delta(x),
$$

then $G(x)=S_{6 k}(x) * R_{4 k}(x) *\left(C^{* k}(x)\right)^{*-1}$ is a Green function for the $\circledast_{B}^{k}$ operator iterated $k$-times where $\circledast_{B}$ is defined by (5), $\delta$ is the Direc delta distribution, $x=\left(x_{1}, x_{2}, \ldots, x_{n}\right) \in \mathbb{R}_{n}^{+}, k$ is a nonnegative integer and

$$
C(x)=-\left[\frac{3}{4} S_{4}(x)+\frac{1}{4} R_{4}(x)\right],
$$

$C^{* k}(x)$ denotes the convolution of $C(x)$ itself $k$-times, $\left(C^{* k}(x)\right)^{*-1}$ denotes the inverse of $C^{* k}(x)$ in the convolution algebra. Moreover $C^{* k}(x)$ is a tempered distribution.

Proof. Since $\circledast_{B}=\frac{3}{4} \diamond_{B} \square_{B}+\frac{1}{4} \triangle_{B}^{3}$, by (9) we obtain

$$
\left[\frac{3}{4} \diamond_{B} \square_{B}+\frac{1}{4} \triangle_{B}^{3}\right]\left[\frac{3}{4} \diamond_{B} \square_{B}+\frac{1}{4} \triangle_{B}^{3}\right]^{k-1} G(x)=\delta(x) .
$$

By Lemma 7 with $k=1, S_{6}(x) * R_{4}(x)$ exists and is a tempered distribution. Convolving both side of the above equation by $S_{6}(x) * R_{4}(x)$, we have

$$
\left[\frac{3}{4} \diamond_{B} \square_{B}+\frac{1}{4} \triangle_{B}^{3}\right]\left(S_{6}(x) * R_{4}(x)\right) *\left[\frac{3}{4} \diamond_{B} \square_{B}+\frac{1}{4} \triangle_{B}^{3}\right]^{k-1} G(x)=\left(S_{6}(x) * R_{4}(x)\right) * \delta(x) .
$$

By Lemma 8, we obtain

$$
C(x) *\left[\frac{3}{4} \diamond_{B} \square_{B}+\frac{1}{4} \triangle_{B}^{3}\right]^{k-1} G(x)=S_{6}(x) * R_{4}(x) .
$$

Keeping on convolving both sides of the above equation by $S_{6}(x) * R_{4}(x)$ up to $k-1$ times, we have

$$
C^{* k}(x) * G(x)=\left(S_{6}(x) * R_{4}(x)\right)^{* k} .
$$


where the symbol $* k$ denotes the convolution of itself $k$-times. By M.A. Tellez (1994), we get

$$
\left(S_{6}(x) * R_{4}(x)\right)^{* k}=S_{6 k}(x) * R_{4 k}(x) .
$$

Therefore,

$$
C^{* k}(x) * G(x)=S_{6 k}(x) * R_{4 k}(x) .
$$

Since $S_{4}(x)$ and $R_{4}(x)$ are lies in $S^{\prime}$ where $S^{\prime}$ is a space of tempered distribution, $C(x) \in S^{\prime}$. By W.F. Donoghue (1996, p. 152), we obtain $C^{* k}(x) \in S^{\prime}$. Since $S_{6 k}(x) * R_{4 k}(x) \in S^{\prime}$, choose $S^{\prime} \subset D_{R}^{\prime}$ where $D_{R}^{\prime}$ is the right-side distribution which is a subspace of $D^{\prime}$ of distribution. Thus $S_{6 k}(x) * R_{4 k}(x) \in D_{R}^{\prime}$, it follows that $S_{6 k}(x) * R_{4 k}(x)$ is an element of the convolution algebra. By A.H. Zemanian (1964, p. 150-151) the equation (11) has an unique solution

$$
G(x)=S_{6 k}(x) * R_{4 k}(x) *\left(C^{* k}(x)\right)^{*-1}
$$

where $\left(C^{* k}(x)\right)^{*-1}$ is an inverse of $C^{* k}(x)$ in the convolution algebra, $G(x)$ is called the elementary solution of the $\circledast_{B}^{k}$ operator. Since $S_{6 k}(x) * R_{4 k}(x)$ and $\left(C^{* k}(x)\right)^{*-1}$ are tempered distribution, by W.F. Donoghue (1996, p. 152), we obtain $S_{6 k}(x) * R_{4 k}(x) *\left(C^{* k}(x)\right)^{*-1}$ is a tempered distribution. It follows that $G(x)$ is a tempered distribution.

Theorem 2. Given the equation

$$
\circledast_{B}^{k} u(x)=f(x)
$$

where $f$ is a given generalized function and $u(x)$ is an unknown function, we obtain

$$
u(x)=G(x) * f(x)
$$

is an unique solution of $(12)$ where $G(x)$ is an elementary solution for the operator $\circledast_{B}^{k}$.

Proof. Convolving both sides of the equation (12) by the Green function $G(x)$ of the $\circledast_{B}^{k}$ operator in Theorem 1 , we obtain

$$
G(x) * \circledast_{B}^{k} u(x)=G(x) * f(x)
$$

or

$$
\circledast_{B}^{k} G(x) * u(x)=G(x) * f(x) .
$$

Applying Theorem 1, we have

$$
\delta(x) * u(x)=G(x) * f(x)
$$

or

$$
u(x)=G(x) * f(x) .
$$

Since $G(x)$ is an unique, $u(x)$ is an unique solution of the equation (12).

\section{Acknowledgment}

This work was granted by Office of the Higher Education Commission. Mr.Somboon Niyom was supported by CHE Ph.D. scholarship and Graduate School, Chiangmai University, Thailand.

\section{References}

A. H. Zemanian. (1964). Distribution Theory and Transform Analysis. Mc-Graw Hill, New York.

A. Kananthai. (1997). On the solution of the n-dimensional Diamond operator. Applied Mathematics and Computation, 88 , p. $27-37$.

B. M. Levitan. (1951). Expansion in Fourier series and integrals with Bessel functions (N.S.). Uspeki Mat. Nauka, 2 (42), p. 102-143 (in Russian).

H. Yildirim. (1995). Riesz Potentials Generated by a Gemeralized Shift Operator. Ph. D. Thesis, Ankara University .

H. Yildirim, M. Z. Sarikaya and S. Öztürk. (2004). The solution of the $n$-dimensional Bessel diamond operator and the Fourier-Bessel transform of their convolution. Proc. Indian Acad. Sci. (Math. Sci.), 114 (4), p. 375-387.

I. M. Gelfand and G. E. Shilov. (1964). Generalized Function. Vol. I, Academic Press, New York.

M. A. Tellez. (1995-96). The convolution product of $W_{\alpha}(u, m) * W_{\beta}(u, m)$. Mathematicae Notae, 38.

M. A. Tellez. (1994). The distribution Hankel transform of Marcel Rieszs ultra-hyperbolic kernel. Studies in Applied Mathematics, 93. 
M. Z. Sarikaya and H. Yildirim. (2009). On the B-convolutions of the Bessel diamond kernel of Riesz. Appl. Math. Comp. 208, p. 18-22.

S. E. Trione. (1987). On Marcel Rieszs ultra-hyperbolic kernel. Trabajos de Math, 116.

W. F. Donoghue. (1969). Distributions and Fourier transforms. Academic Press, New York.

W.Satsanit, A.Kananthai.(2010). On the operator $\circledast^{k}$ Relate to Heat Equation. Journal of Mathematics Research, Vol.2, No.2, p.20-27. 